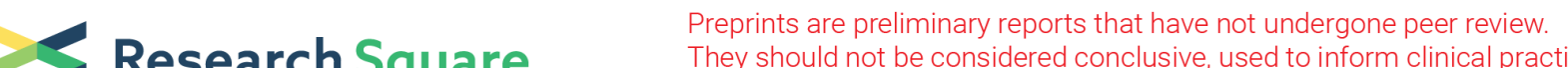 $\begin{array}{ll}\text { Research Square } & \text { They should not be considered conclusive, used to inform clinical practice, } \\ \text { or referenced by the media as validated information. }\end{array}$
}

\section{ALAD Expression and Prognostic Value in Multiple Human Cancers: A Bioinformatics Analysis}

\section{Quan Chen}

Guangzhou Medical University Second Affiliated Hospital

\section{Chao Jiang}

People's hospital of Shenzhen Baoan District

Jing Li

Guangzhou Medical University

\section{Pei Qiong Li}

Guangzhou Medical University

\section{Ye Kui Yin}

Guangzhou Medical University

\section{Qing Nan Liu}

Guangzhou Medical University

Xiaohui Zhao ( $\nabla$ xiaohuizhao27@163.com )

Guangzhou Medical University https://orcid.org/0000-0002-5851-208X

\section{Primary research}

Keywords: ALAD, data mining, enrichment analysis, molecular targeted therapy, prognostic biomarker

Posted Date: December 28th, 2020

DOl: https://doi.org/10.21203/rs.3.rs-132679/v1

License: (c) (i) This work is licensed under a Creative Commons Attribution 4.0 International License.

Read Full License 


\section{Abstract}

Background: $\delta$-aminolevulinic acid dehydratase (ALAD) is a kind of metalloenzyme, which can catalyze $\delta$ aminolevulinic acid to synthesize bilirubin. However, the expression level, prognostic role, biological function and mechanism of action of ALAD in a variety of human tumors are still not clear.

Methods: GEPIA Online (http://gepia.cancer-pku.cn) website was used for mRNA analysis of ALAD in multiple human solid tumors. NCBI/GEO database and UALCAN website (http://ualcan.path.uab.edu) were used to analyze the ALAD mRNA expression in breast cancer and lung cancer. Immunohistochemical (IHC) analysis from HPA database was used to detect the protein expression of ALAD in different human tumors. Kaplan-Meier plotter database (http://kmplot.com/analy sis/) was used to evaluate the prognostic value of ALAD expression in different human tumors. Gene Ontology (GO) analysis, Kyoto Encyclopedia of Gene and Genome (KEGG) pathway analysis and Gene Set Enrichment Analysis (GSEA) analysis were used for ALAD enrichment analysis and signal pathway analysis.

Results: Analyzed by GEPIA, UALCAN online website and GEO analysis in multiple human tumors, the results showed that ALAD mRNA expression is significantly down-regulated and positively correlated with the overall survival (OS) time of different cancer patients. Besides, the expression of ALAD protein is always none or low in multiple solid tumors. In addition, GO and KEGG enrichment analysis suggested that ALAD may play an anti-tumor role in many directions, and GSEA further found that the overexpression of ALAD is negatively correlated with cell cycle signal pathway and PI3K/AKT/mTOR signal pathway.

Conclusions: In conclusion, our study demonstrated for the first time that ALAD is downregulated in multiple human cancers and ALAD low expression is associated with worse overall survival of different cancer patients. Furthermore, we showed that ALAD low expression is associated with tumor cell cycle process and $\mathrm{PI} 3 \mathrm{~K} / \mathrm{AKT} / \mathrm{mTOR}$ signaling pathway. ALAD may be a valuable prognostic biomarker and therapeutic target of multiple human tumors.

\section{Background}

With the continuous deepening and development of human genomics, pharmacogenomics and tumor biology, the diagnosis and treatment of cancer has gradually stepped into the era of molecular level. Nowadays, the difference between tumor and normal cells can be found through its special "molecular characteristics" in the early stage of malignant tumor [1], including oncogene, tumor suppressor, gene mutation and chromatin modification. Based on this, tumor molecular targeting therapy becomes increasingly important. It takes specific molecules of tumors as the therapeutic target, and specific drugs aimed at these specific molecules are used to kill tumor cells and destroy their growth environment with little or no damage to normal tissues and cells as much as possible. In recent years, great progress has been made in the field of tumor targeted therapy, such as in the identification of new tumor antigens [2, 3], in the mechanism studies [4-6], and in the optimization of antibody structure [7-9]. At present, the 
molecular targeted therapy has showed definite effects in clinical practice: targeted therapy combined with radiotherapy can strongly inhibit the expression of COX-2 and VEGF in bone metastasis of lung cancer [10]; HRG is a new target for ER positive breast cancer [11]; sorafenib, a multi-target kinase inhibitors, is the preferred choice for advanced liver cancer [12]; and imatinib, a receptor tyrosine kinase inhibitor, is effective for chronic myeloid leukemia [13]. The precondition of employing tumor targeted therapy is to accurately evaluate the expression of tumor molecular targets that can be used as therapeutic targets and biological markers of the disease and predict the biological characteristics of the disease.

ס-aminolevulinic acid dehydratase (ALAD), a homomeric metallomeric enzyme that catalyzes 5aminolevulinic acid to produce bilirubin, is located on human chromosome 9q34 [14]. The active site of ALAD has the common characteristics of both metal aldolase and Schiff base aldolase, so ALAD represents an intriguing combination of both classes of enzyme. Generally, ALAD is believed to be closely related to lead. On the one hand, lead ions can replace the zinc bound to the enzyme and effectively inhibit ALAD [15], and besides, lead exposure increases the abnormal methylation of ALAD [16]. On the other hand, the activity of ALAD can be used as a biomarker of environmental lead exposure and toxicity in prokaryotes and eukaryotes [17]. More importantly, it is reported that ALAD has been shown to be associated with the risk of a variety of cancers. For example, common genetic mutations in ALAD may change the overall risk of renal cell carcinoma [18]; genetic variation of ALAD may change the association between lead and prostate cancer [19]; single nucleotide polymorphisms in the ALAD gene affect the association between lead exposure and brain tumor risk [20]. Besides, it has also been reported that ALAD- proteasome complexes may have a clinical impact on improving anticancer therapy depended on HDAC inhibitors [21]. Based on the above reports, it is an attractive research direction to study whether ALAD can be used as a molecular targets in a series of human solid tumors.

In this study, TCGA database and GEPIA analysis showed that ALAD mRNA expression was significantly down-regulated in BRCA, ESCA, KIRC, KIRP, LUAD, LUSC, THYM and UCEC cancer tissues. Besides, GEO database and UALCAN website indicated that the mRNA expression of ALAD in BRCA, LUAD and LUSC cancer tissues was significantly reduced compared with adjacent normal tissues. In addition, HPA database suggested that ALAD protein was not expressed or low expressed in different tumors. At the same time, KM plotter analysis showed that enhanced ALAD expression was associated with a more favorable outcome of overall survival time in patients with BRCA, ESCA, KIRC, KRIP, LIHC, LUAD, LUSC, SARC, THYM and UCEC. These findings suggest that ALAD may be a potential biomarker for predicting the prognosis of many human cancers. Further, GO enrichment analysis showed that ALAD had significant effects on the negative regulation of transcription from RNA polymerase II promoter and positive regulation of cell proliferation, and KEGG pathway enrichment analysis suggested that ALAD mainly involved in cancer pathways. Mechanistically, GSEA analysis indicated that upregulation of ALAD might inhibit cell cycle and PI3K/AKT/mTOR signaling pathway. Taken together, all the above findings suggest that ALAD may be a potential biomarker for predicting the prognosis of a variety of human cancers and play a tumor inhibitory role in human breast cancer and lung cancer. 


\section{Materials And Methods}

\section{TCGA Dataset Analysis}

Gene Expression Profiling Interactive Analysis (GEPIA) online (http://gepia.cancer-pku.cn/) is a newly developed interactive web server for analyzing the RNA sequencing expression data of 9736 tumors and 8587 normal samples from the TCGA and the GTEx projects [22]. In this study, GEPIA database, being presented in Expression DIY-box plots (in the 'Boxplot' tab) and containing ten dataset selections which are matched normal TCGA data for analysis, was used to analyze expression levels of ALAD and IncRNAs in them. Genes with $|\log 2 \mathrm{FC}|>1$ and $p$-value $<0.05$ were considered as statistically significant. TCGA Study Abbreviations are shown in Table 1.

\section{Gene Expression Omnibus Expression analysis}

The Gene Expression Omnibus (GEO, http://www.ncbi.nlm.nih.gov/geo/) is an international public repository for high-throughput microarray and next-generation sequence functional genomic data sets submitted by the research community and all data are freely available for download in a variety of formats [23]. Four independent breast cancer and lung cancer gene expression profiles (GSE5364, GSE134359, GSE33532, and GSE56044) were downloaded from GEO database and these datasets were obtained from the microarray platform of Affymetrix Human Genome U133A Array [HG-U133A], Affymetrix Human Transcriptome Array 2.0 [transcript (gene) version] [HTA-2_0], Affymetrix Human Genome U133 Plus 2.0 Array [HG-U133_Plus_2] and Illumina HumanMethylation450 BeadChip (HumanMethylation450_15017482). After that, the gene chips and sequencing results was used to analyze the expression level of ALAD mRNA in cancer tissues and adjacent normal tissues in breast invasive carcinoma (BRCA), lung adenocarcinoma (LUAD) and lung squamous cell carcinoma (LUSC).

\section{UALCAN Online Expression analysis}

UALCAN (http://ualcan.path.uab.edu) is used to construct an algorithm based on the TCGA level 3 RNAseq database and all data are divided into 31 types according to the type of cancer. It can be used to analyze relative transcriptional expression of potential genes of interest between tumor and normal samples and association of the transcriptional expression with relative clinicopathologic parameters [24]. In this study, UALCAN was used to analyze the mRNA expression of ALAD in BRCA, LUAD and LUSC dataset from TCGA, including total expression, sex, age, race, tumor stage, lymph node metastasis and TP53 gene mutation.

\section{Immunohistochemical analysis}

The expression of ALAD protein was analyzed by the HPA database (https://www.proteinatlas.org/). ALAD was entered into the database to obtain the ALAD expression level of a single tumor of each cancer type (scar strip $=200 \mu \mathrm{m}$ ). All IHC images have been manually annotated by a certified pathologist $[25$, 26]. 


\section{Prognostic analysis}

The Kaplan-Meier plotter (http://kmplot.com/analysis/) is an online tool applied to assess the effect of 54,675 genes on survival using 10,461 cancer samples (5,143 breast, 1,816 ovarian, 2,437 lung, and 1,065 gastric cancer) [27]. Online KM plotter database based on TCGA database is used to analyze the relationship between a specific gene in tumor and patients' survival time of death. The results are shown by $95 \%$ confidence interval and risk-to-risk ratio.

\section{Enrichment analysis}

The LinkedOmics database (http://www.linkedomics.org/login.php) was used to look for the differentially expressed genes (DEGs) related to ALAD. We analyzed methylation data by search dataset and target dataset using Spearman test in TCGA-BRCA dataset, TCGA-LUAD dataset and TCGA-LUSC dataset, to work out the correlation coefficient between DEGs and ALAD (Without subset database). Then, the selected genes were analyzed by the Database for Annotation, Visualization, and Integrated Discovery (DAVID, https://david.ncifcrf.gov/home.jsp) for Gene Ontology (GO) analysis and Kyoto Encyclopedia of Gene and Genome (KEGG) pathway enrichment analysis to get the results. Based on the enrichment theory of go and KEGG pathways, we encode each essential / nonessential gene into a vector, where each component represents the relationship between the gene and a go term or KEGG pathway [28]. Eventually, those results were visualized by bioinformatics online tool (http://www.bioinformatics.com.cn).

\section{Correlation analysis}

Gene Set Enrichment Analysis (GSEA) is routinely used to analyze and interpret coordinate pathway-level changes in transcriptomics experiments [29]. Besides, GSEA is a computational method that assesses whether an a priori defined set of genes shows statistically significant, concordant differences between two biological states [30]. GSEA was used to analyze the relationship between ALAD expression and cell cycle signal pathway and PI3K/AKT signaling pathway in patients with breast cancer and lung cancer.

\section{Statistical analysis}

GraphPad PRISM 5 software (GraphPad Software, San Diego, California, USA) was used for all statistical analyses. Differences between two groups were analyzed using the two-tailed unpaired Student's $t$-test; $P<0.05$ was considered statistically significant.

\section{Results}

\section{ALAD mRNA Expression Is Down-regulated in Ten Solid Tumor Types}

To determine the potential role of ALAD in a variety of human cancers, we first verified the expression level of ALAD mRNA using the TCGA database and GEPIA analysis. The results showed that ALAD mRNA 
expression was significantly down-regulated in breast invasive carcinoma (BRCA), esophageal cancer (ESCA), renal clear cell carcinoma (KIRC), renal papillary cell carcinoma (KIRP), lung adenocarcinoma (LUAD), lung squamous cell carcinoma (LUSC), thymoma (THYM) and endometrial carcinoma (UCEC). In liver hepatocellular carcinoma (LIHC) and sarcoma (SARC), although the results were not statistically significant, the average expression level of ALAD mRNA in cancer tissues was lower than that in normal tissues (Fig. 1).

\section{ALAD mRNA Expression Is Low in BRCA, LUAD and LUSC}

By downloading the microarray results related to breast cancer and lung cancer from the NCBI/GEO public database, we found ALAD mRNA expression was obviously down-regulated in cancer tissues compared to adjacent normal tissues in the GSE5364 (ANT = 13; $T=83$ ), the GES134359 (ANT = 12; $\mathrm{T}=$ 74), the GSE33532 (ANT = 20; $T=80$ ) and the GSE56044 (ANT = 12; $T=124$ ) (Fig. 2). Moreover, by analysis in UALCAN website, we found the mRNA expression of ALAD was higher in the normal samples (BRCA group $n=114$; LUAD group $n=59$; LUSC group $n=52$ ) than in the cancer samples (BRCA group $n=$ 1097; LUAD group $n=515$; LUSC group $n=503$; Fig. 3A, 3H, 30). Further, subgroup analysis showed that ALAD was also significantly down-regulated in different subgroups of BRCA, LUAD, and LUSC, including gender, age, and race subgroups (Fig. 3B-D, 3I-K, and 3P-R). Regarding to cancer stages, whether in BRAC, LUAD or in LUSC, we found that ALAD mRNA expression in stage 1-4 was yet lower than that in normal tissues (Fig. 3E, 3L, and $3 S$ ). In view of lymph node metastasis - $N$ staging, ALAD mRNA expression was also significantly lower in cancer tissues with NO-N3 stages than that in normal tissues (Fig. 3F, 3M, and 3T). In addition, ALAD mRNA expression was significantly decreased in BRCA and LUAD tumors with TP53 mutation than that without TP53 mutation (Fig. 3G and 3N). Through all the above analysis, we suggest that ALAD may play a tumor inhibitory role in the progression of breast cancer and lung cancer.

\section{ALAD Protein Expression Is Down-regulated in Ten Solid Tumor Types}

The protein expression of ALAD was analyzed using the HPA database in different tumor types. The results showed that ALAD was low expressed and/or absent in breast cancer (91\%), renal cancer (91\%), lung cancer (89\%), endometrial cancer (83\%), urothelial cancer (100\%), ovarian cancer $(91 \%)$, gastric cancer $(100 \%)$, prostate cancer $(91 \%)$, cervical cancer (100\%) and colorectal cancer $(83 \%)$. However, there are exceptions in liver cancer (42\%) and thyroid cancer (50\%) whose ALAD protein expression level are not over $50 \%$ (Fig. 4A-B). These results suggest ALAD may play a tumor inhibitory role in most tumors.

\section{Alad High Expression Is Associated With Better Overall Survival}

To further study the prognostic role of ALAD in different human cancers, KM plotter database was used to evaluate the overall survival time which was calculated according to the high and low expression of 
ALAD. The results showed that the overall survival time is longer in ALAD high expression group than in ALAD low expression group in BRCA ( $\mathrm{HR}=0.79 ; P=0.029)$, ESCA $(\mathrm{HR}=0.37 ; P=0.0058), \mathrm{KIRC}(\mathrm{HR}=0.42$; $P=5.5 \mathrm{e}-07), \operatorname{KIRP}(\mathrm{HR}=0.42 ; P=0.0041), \mathrm{LIHC}(\mathrm{HR}=0.51 ; P=8.8 \mathrm{e}-05), \mathrm{LUAD}(\mathrm{HR}=0.67 ; P=6 \mathrm{e}-04), \mathrm{LUSC}$ $(\mathrm{HR}=0.45 ; P=0.029), \mathrm{SARC}(\mathrm{HR}=0.56 ; P=0.0035)$, THYM $(\mathrm{HR}=0.12 ; P=0.016)$ and UCEC $(\mathrm{HR}=0.53 ; P$ $=0.003$; Fig. 5), and statistical analysis suggested that the $p$ value is less than 0.05 in all the above ten tumors. These results indicate that ALAD may act as a tumor suppressor and may be a valuable biomarker for predicting prognosis of patients with BRCA, ESCA, KIRC, KIRP, LIHC, LUAD, LUSC, SARC, THYM and UCEC.

\section{Alad Enrichment Analysis Of Brca, Luad And Lusc}

From LinkedOmics website and based on the Spearman test, we screened the differentially expressed genes (DEGs) changed with ALAD expression level in BRCA, LUAD and LUSC (Fig. 6A, 6D, 6G). The first 50 positively related genes and the first 50 negatively related genes in different tumors were shown in the form of heat map (Fig. 6B-C, 6E-F, 6H-I). Moreover, 561 DEGs were screened by Spearman test more than 0.2 in the BRCA dataset; 1401 DEGs were screened by Spearman test more than 0.4 in LUAD dataset; 1910 DEGs were screened by Spearman test more than 0.4 in LUSC dataset. Among all the above DEGs, 542 DEGs overlap each other (Fig. 7A). GO and KEGG enrichment analysis on the DAVID website obtains the relevant data about Biological Process (BP), Cell Composition (CC), Molecular Function (MF) and KEGG pathway. The results are visualized by bioinformatics online tools. For the BP, the negative regulation of transcription from RNA polymerase II promoter, positive regulation of cell proliferation and cell proliferation were the top three relevant pathways (Fig. 7B). For the $\mathrm{CC}$, the potential target genes were predominantly enriched in the cytoplasm, cytoso and membrane (Fig. 7C). For the MF, the mainly significantly involved items were 
protein binding (Fig. 7D). Furthermore, KEGG analysis showed that cancer pathways, proteoglycans in cancer, Wnt signaling pathways and pathways regulating pluripotency of stem cells were the main pathways related to ALAD regulation (Fig. 7E). Collectively, the above results show that ALAD may be involved in many regulation processes in tumors and may be a hopeful therapy target. More studies are needed to further investigate its functions and regulation mechanism.

ALAD Expression Is Negatively Correlated with Cell Cycle and PI3K/AKT/mTOR Signaling Pathway

To more deeply explore the regulation mechanism of ALAD, we conducted GSEA analysis and the results revealed that ALAD expression negatively correlated with the cell cycle characteristics (HALLMARK_G2M_CHECKPOINT) in the publicly available GEO breast cancer database (NCBI/GEO/GES5364, $n=341$ ) and lung cancer database (NCBI/GEO/GSE33532, $n=100$ ) (Fig. 8A). To understand the mechanism of ALAD-mediated cell cycle in breast cancer and lung cancer, we used the same GEO databases to study the relationship between ALAD expression levels and the cancer signaling pathways which closely related to cell cycle regulation, and we found that ALAD expression was negatively correlated with the PI3K/AKT/mTOR pathway (HALLMARK_PI3K_AKT_MTOR_SIGNALING) (Fig. 8B). The above results suggest that ALAD may inhibit the cell cycle process and the PI3K/AKT/mTOR signaling pathway and consequently suppress the cancer cells growth and proliferation. 


\section{Discussion}

ALAD, involved in heme biosynthesis process, is an endogenous inhibitor of 265 proteasome and a target of cancer therapy [31,32]. It has been reported that the gene level and protein level of ALAD are low in breast cancer cell lines, and ALAD plays a tumor inhibitory role in the progression of breast cancer [33]. In addition, Ge et al. point out that ALAD can inhibit the EMT process induced by TGF- $\beta$ and reduce the invasive ability of cancer cells, suggesting that ALAD may be a potential tumor suppressor.

In this study, we selected ten human solid tumors in TCGA database to compare the expression of ALAD mRNA in cancer tissues and normal tissues. The results showed that ALAD mRNA was significantly lower in BRCA, ESCA, KIRC, KIRP, LUAD, LUSC, THYM and UCEC compared with normal tissues, suggesting that ALAD mRNA is under-expressed in most solid tumors. Secondly, to explore the anti-function roles of $A L A D$, we selected the breast cancer and lung cancer for further data mining. The results from NCBI/GEO public database showed that the cancer groups had a significantly lower level of ALAD expression than the control groups in GSE5364, GSE134359, GSE33532 and GSE56044 datasets. Besides, the UALCAN database showed that ALAD mRNA expression levels inversely correlated with sex, age, race, tumor stage, regional lymph node metastasis and TP53 mutation. IHC results from HPA database showed that ALAD protein was not expressed or under-expressed in most human solid tumors. In addition, the patients with high ALAD had a longer overall survival time than the ones with low ALAD, and the $p$ values of the ten Kaplan-Meier survival curves were all less than 0.05, suggesting that high expression of ALAD indicates a better prognosis. Taken together, these results support that ALAD may be a tumor suppressor gene in human solid tumors and an important biomarker for predicting the survival of cancer patients.

To more systematically explain the biological functions and molecular mechanism of ALAD and find signal pathways involved in the regulation of $A L A D$, we conducted enrichment analysis. $G O$ analysis showed that the differentially expressed genes (DEGs) related to ALAD were mainly concentrated in the negative regulation of RNA polymerase II promoter transcription, cell proliferation and protein binding, while KEGG analysis showed that these DEGs were mainly concentrated in cancer pathways and Wnt signaling pathway. This suggests that ALAD has a wide range of biological functions. However, what is the regulation mechanism of ALAD in these tumors? Aiming to solve this problem, we conducted GSEA analysis to screen the signal pathways closely correlated with the regulation of ALAD. The results showed that cell cycle (HALLMARK_G2M_CHECKPOINT) regulation was negatively correlated with ALAD expression in breast cancer and lung cancer, suggesting that ALAD may inhibit the tumor progression by inhibiting the cell cycle process. Next, we used the same method to further study the mechanism of action of $A L A D$, and the results showed that ALAD expression was negatively correlated with $\mathrm{PI3K} / \mathrm{AKT} / \mathrm{mTOR}$ signaling pathway (HALLMARK_PI3K_AKT_MTOR_SIGNALING) which can inhibit tumor growth, proliferation, migration and invasion. The combination of the two indicates that ALAD may inhibit tumor cells growth, proliferation, migration and invasion by inhibiting the cell cycle process and the PI3K/AKT/mTOR pathway. Collectively, we believe that ALAD can be used as a molecular target in the diagnosis and treatment of cancers. 


\section{Conclusion}

After in-depth data mining of ALAD, we found that the expression of ALAD in cancer tissues was downregulated compared with normal tissues, and ALAD high expression is associated with better overall survival of cancer patients. The mechanism may be that ALAD can regulate the cell cycle process and $\mathrm{PI} 3 \mathrm{~K} / \mathrm{AKT} / \mathrm{mTOR}$ signal pathway. In conclusion, ALAD may be a valuable prognosis biomarker and hopeful therapeutic target for multiple human tumors.

\section{Abbreviations}

ALAD: $\delta$-aminolevulinic acid dehydratase; NCBI/GEO: The Gene Expression Omnibus; TCGA: The Cancer Genome Atlas; IHC: Immunohistochemical; HPA: Human Pathological Atlas; KM plotter: Kaplan-Meier plotter; OS: Overall Survival; DAVID: the Database for Annotation, Visualization, and Integrated Discovery; GO: Gene Ontology; KEGG: Kyoto Encyclopedia of Gene and Genome; BP: Biological Process; CC: Cell Composition; MF: Molecular Function; GSEA: Gene Set Enrichment Analysis; HDAC: Histone deacetylase; COX-2: Cyclooxygenase-2; VEGF: Vascular Endothelial-derived Growth Factor; HRG: Host-produced histidine-rich Glycoprotein.

\section{Declarations}

\section{Authors' contributions}

CQ and ZXH designed the study, prepared, edited and reviewed the manuscript. CQ, JC, LJ, LPQ, YYK, LQN and ZXH did literature research, gave comments and reviewed the manuscript. CQ, JC and ZXH designed the study and wrote the manuscript. All authors read and approved the final manuscript.

\section{Acknowledgments}

Not applicable

\section{Competing interests}

The authors declare that they have no competing interests.

\section{Availability of data and materials}

All data generated or analyzed during this study are included in this published article.

\section{Consent for publication}

All authors approved the publication of this manuscript.

\section{Ethical approval and consent to participate}


All the procedures in this study involving human participants were carried out following the 1964 Helsinki declaration and its subsequent amendments.

\section{Funding}

This work was supported by Guangzhou Medical University High-level University Academic Key Cultivation Program (Grant No. B185004137); the youth innovative talents program of regular institutions of higher education of Guangdong Province (Grant No. 2018KQNCX212).

\section{References}

1. Cancer Genome Atlas Research. N., Comprehensive genomic characterization of squamous cell lung cancers. Nature. 2012;489(7417):519-25.

2. Sigismund S, Avanzato D, Lanzetti L. Emerging functions of the EGFR in cancer. Mol Oncol. 2018;12(1):3-20.

3. Anderson DJ, et al. Targeting the AAA ATPase p97 as an Approach to Treat Cancer through Disruption of Protein Homeostasis. Cancer Cell. 2015;28(5):653-65.

4. Spranger S, et al., Tumor-Residing Batf3 Dendritic Cells Are Required for Effector T Cell Trafficking and Adoptive T Cell Therapy. Cancer Cell, 2017. 31(5): 711-23 e4.

5. Risom T, et al. Deregulating MYC in a model of HER2 + breast cancer mimics human intertumoral heterogeneity. J Clin Invest. 2020;130(1):231-46.

6. Liu P, et al. Crizotinib-induced immunogenic cell death in non-small cell lung cancer. Nat Commun. 2019;10(1):1486.

7. Morrison C. Nanobody approval gives domain antibodies a boost. Nat Rev Drug Discov. 2019;18(7):485-7.

8. Duchardt-Ferner E, et al. Structure of an RNA aptamer in complex with the fluorophore tetramethylrhodamine. Nucleic Acids Res. 2020;48(2):949-61.

9. Liu C, et al. Preparation of single-chain Fv antibodies in the cytoplasm of Escherichia coli by simplified and systematic chaperone optimization. J Biochem. 2019;166(6):455-62.

10. Hu Y, et al. Effect of molecular targeted therapy combined with radiotherapy on the expression and prognostic value of COX-2 and VEGF in bone metastasis of lung cancer. J BUON. 2020;25(2):81120.

11. Fukui F, Hayashi SI, Yamaguchi Y. Heregulin controls ERalpha and HER2 signaling in mammospheres of ERalpha-positive breast cancer cells and interferes with the efficacy of molecular targeted therapy. J Steroid Biochem Mol Biol. 2020;201:105698.

12. Helmy KY, et al. Cancer immunotherapy: accomplishments to date and future promise. Ther Deliv. 2013;4(10):1307-20.

13. Llovet JM, et al. Molecular therapies and precision medicine for hepatocellular carcinoma. Nat Rev Clin Oncol. 2018;15(10):599-616. 
14. Wetmur JG, et al. Molecular characterization of the human delta-aminolevulinate dehydratase 2 (ALAD2) allele: implications for molecular screening of individuals for genetic susceptibility to lead poisoning. Am J Hum Genet. 1991;49(4):757-63.

15. Erskine PT, et al. X-ray structure of 5-aminolaevulinate dehydratase, a hybrid aldolase. Nat Struct Biol. 1997;4(12):1025-31.

16. Yohannes YB, et al. Blood lead levels and aberrant DNA methylation of the ALAD and p16 gene promoters in children exposed to environmental-lead. Environ Res. 2020;188:109759.

17. Cigerci $\mathrm{IH}$, et al. Comparison of ALAD activities of Citrobacter and Pseudomonas strains and their usage as biomarker for Pb contamination. Environ Monit Assess. 2008;139(1-3):41-8.

18. van Bemmel DM, et al. Comprehensive analysis of 5-aminolevulinic acid dehydrogenase (ALAD) variants and renal cell carcinoma risk among individuals exposed to lead. PLoS One. 2011;6(7):e20432.

19. Neslund-Dudas $C$, et al. Case-only gene-environment interaction between ALAD tagSNPs and occupational lead exposure in prostate cancer. Prostate. 2014;74(6):637-46.

20. Bhatti $P$, et al. Comparison of occupational exposure assessment methods in a case-control study of lead, genetic susceptibility and risk of adult brain tumours. Occup Environ Med. 2011;68(1):4-9.

21. Schmitt SM, et al. Involvement of ALAD-20S Proteasome Complexes in Ubiquitination and Acetylation of Proteasomal alpha2 Subunits. J Cell Biochem. 2016;117(1):144-51.

22. Tang Z, et al. GEPIA: a web server for cancer and normal gene expression profiling and interactive analyses. Nucleic Acids Res. 2017;45(W1):W98-102.

23. Barrett T, et al. NCBI GEO: archive for functional genomics data sets-update. Nucleic Acids Res. 2013;41(Database issue):D991-5.

24. Chandrashekar DS, et al. UALCAN: A Portal for Facilitating Tumor Subgroup Gene Expression and Survival Analyses. Neoplasia. 2017;19(8):649-58.

25. Zhang J, et al. Epigenetic-Mediated Downregulation of Zinc Finger Protein 671 (ZNF671) Predicts Poor Prognosis in Multiple Solid Tumors. Front Oncol. 2019;9:342.

26. Uhlen M, et al., A pathology atlas of the human cancer transcriptome. Science, 2017. 357(6352).

27. Li MX, et al. Identification of potential core genes in triple negative breast cancer using bioinformatics analysis. Onco Targets Ther. 2018;11:4105-12.

28. Chen $L$, et al. Prediction and analysis of essential genes using the enrichments of gene ontology and KEGG pathways. PLoS One. 2017;12(9):e0184129.

29. Powers RK, et al. GSEA-InContext: identifying novel and common patterns in expression experiments. Bioinformatics. 2018;34(13):i555-64.

30. Subramanian A, et al. GSEA-P: a desktop application for Gene Set Enrichment Analysis. Bioinformatics. 2007;23(23):3251-3.

31. Guo GG, Gu M, Etlinger JD. 240-kDa proteasome inhibitor (CF-2) is identical to delta-aminolevulinic acid dehydratase. J Biol Chem. 1994;269(17):12399-402. 
32. Bardag-Gorce F, French SW. Delta-aminolevulinic dehydratase is a proteasome interacting protein. Exp Mol Pathol. 2011;91(2):485-9.

33. Ge J, et al. Downregulation of delta-aminolevulinate dehydratase is associated with poor prognosis in patients with breast cancer. Cancer Sci. 2017;108(4):604-11.

\section{Tables}

TCGA Study Abbreviations

\begin{tabular}{|ll|}
\hline Study Abbreviation & Study Name \\
\hline BRCA & Breast invasive carcinoma \\
\hline ESCA & Esophageal carcinoma \\
\hline KIRP & Kidney renal clear cell carcinoma \\
\hline LIHC & Kidney renal papillary cell carcinoma \\
\hline LUAD & Liver hepatocellular carcinoma \\
\hline LUSC & Lung adenocarcinoma \\
\hline SARC & Lung squamous cell carcinoma \\
\hline THYM & Sarcoma \\
\hline UCEC & Thymoma \\
\hline
\end{tabular}

\section{Figures}


Figure 1
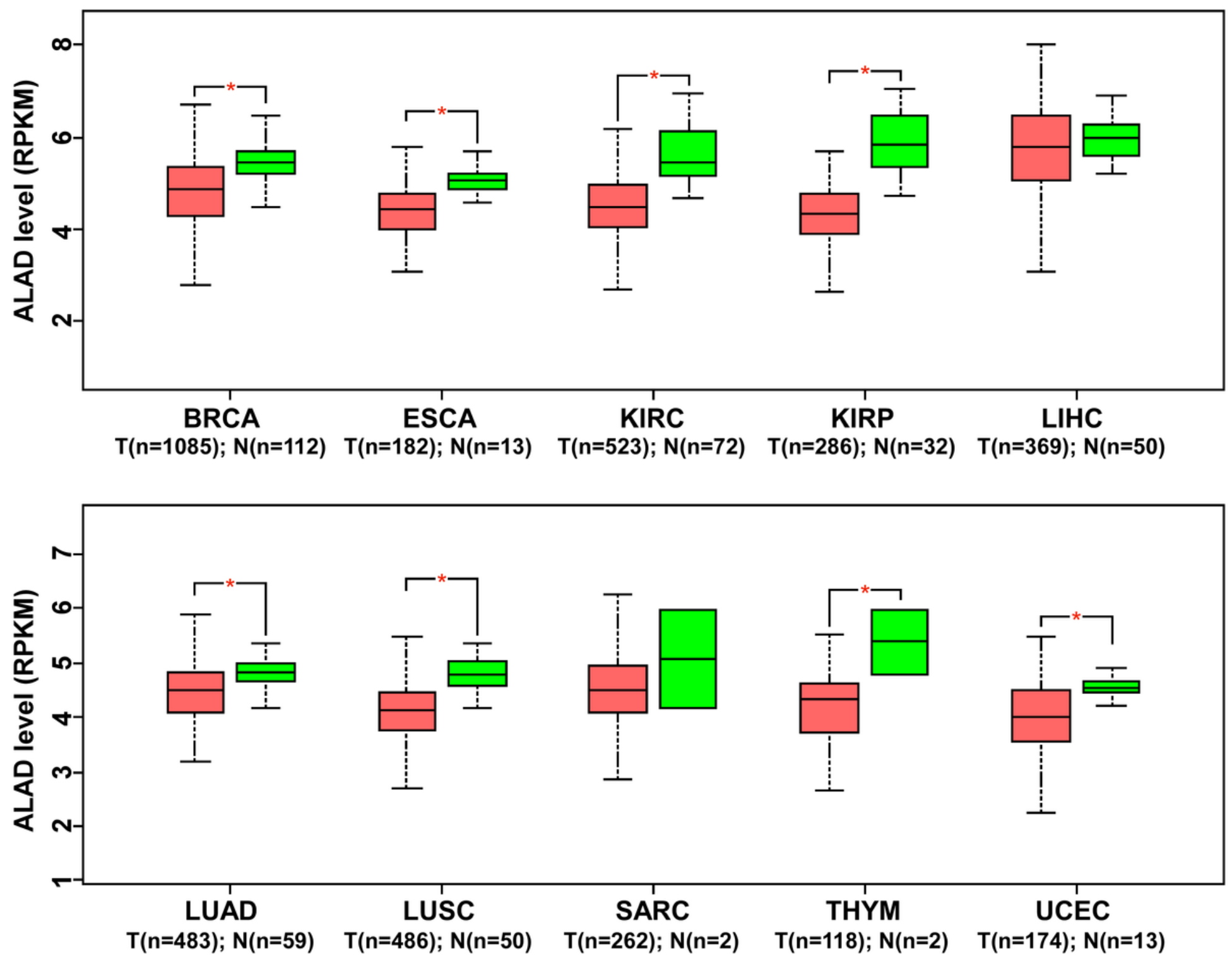

Figure 1

Based on TCGA dataset, ALAD mRNA expression is downregulated in multiple human cancers by GEPIA analysis. * $P<0.05$. Abbreviations: BRCA, Breast invasive carcinoma; ESCA, Esophageal cancer; KIRC, Kidney renal clear cell carcinoma; KIRP, Kidney renal papillary cell carcinoma; LIHC, Liver hepatocellular carcinoma; LUAD, Lung Adenocarcinoma; LUSC, Lung squamous Cell carcinoma; SARC, Sarcoma; THYM, Thoracic Adenocarcinoma; UCEC, Uterine Corpus Endometrial Carcinoma. 
Figure 2

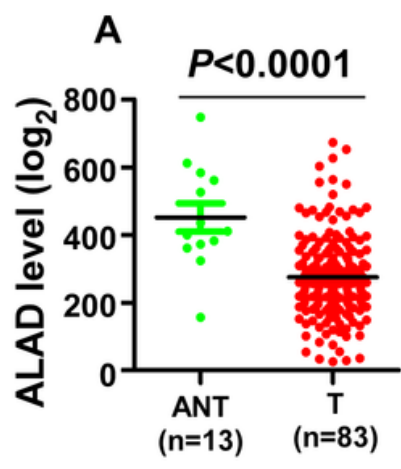

GSE5364

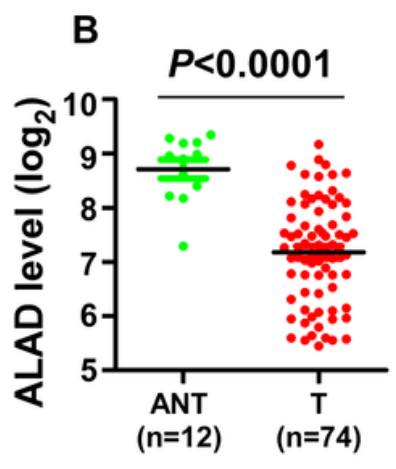

GSE134359

ALAD expression in Breast Cancer

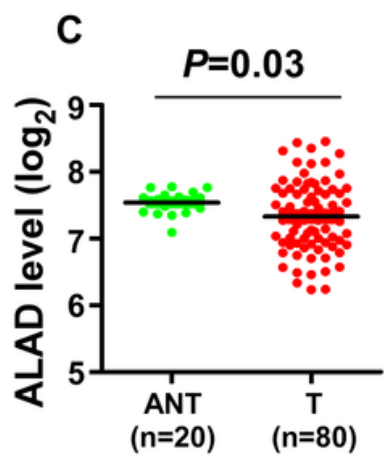

GSE33532

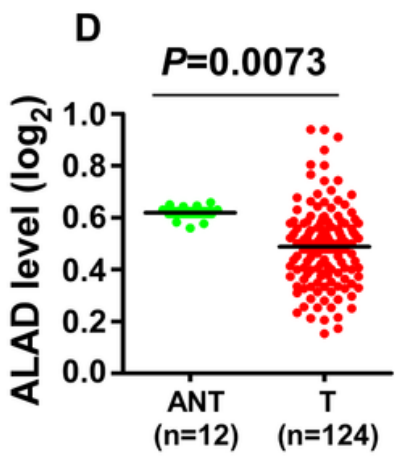

GSE56044

ALAD expression in Lung Cancer

Figure 2

ALAD gene microarrays in breast cancer and lung cancer in NCBI/GEO public database. (A-B) ALAD mRNA expression results in breast cancer GSE5364 (ANT=13; T=83) and GSE134359 (ANT=12; T=74) datasets. (C-D) ALAD mRNA expression results in lung cancer GSE33532 (ANT=20; $T=80$ ) and GSE56044 (ANT $=12 ; T=124$ ) datasets. 
Figure 3
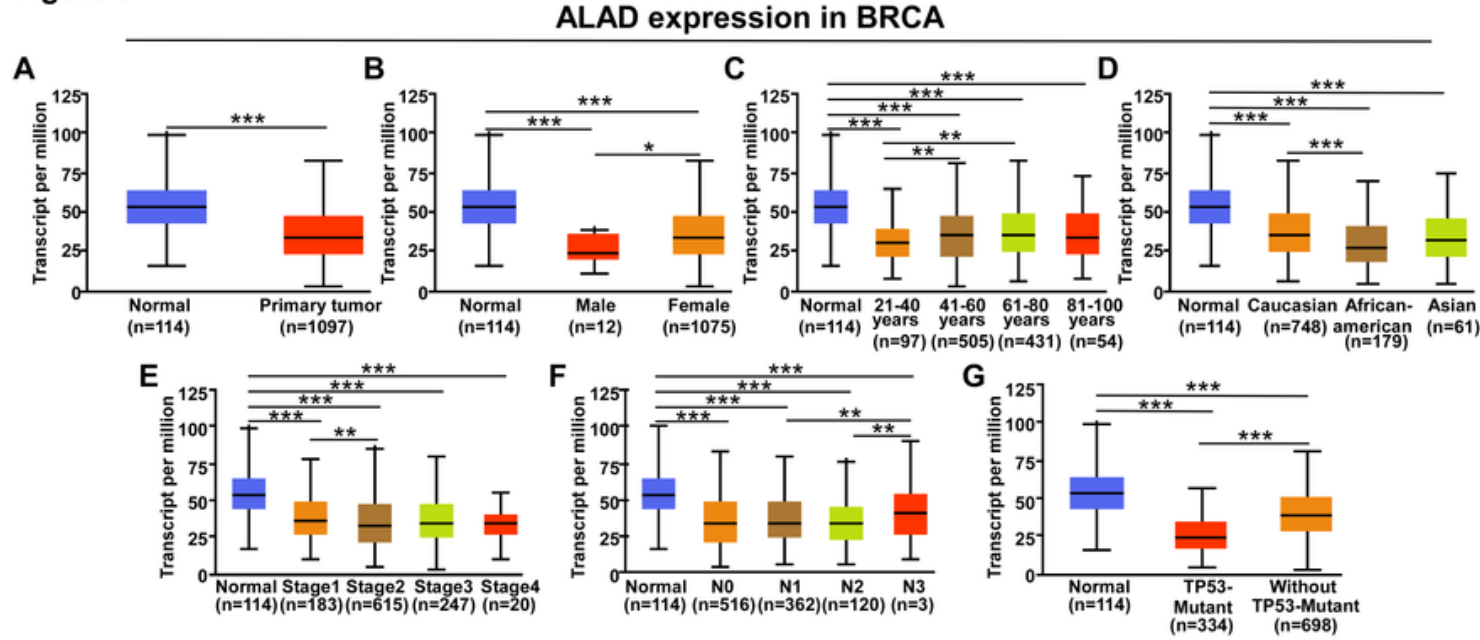

ALAD expression in LUAD

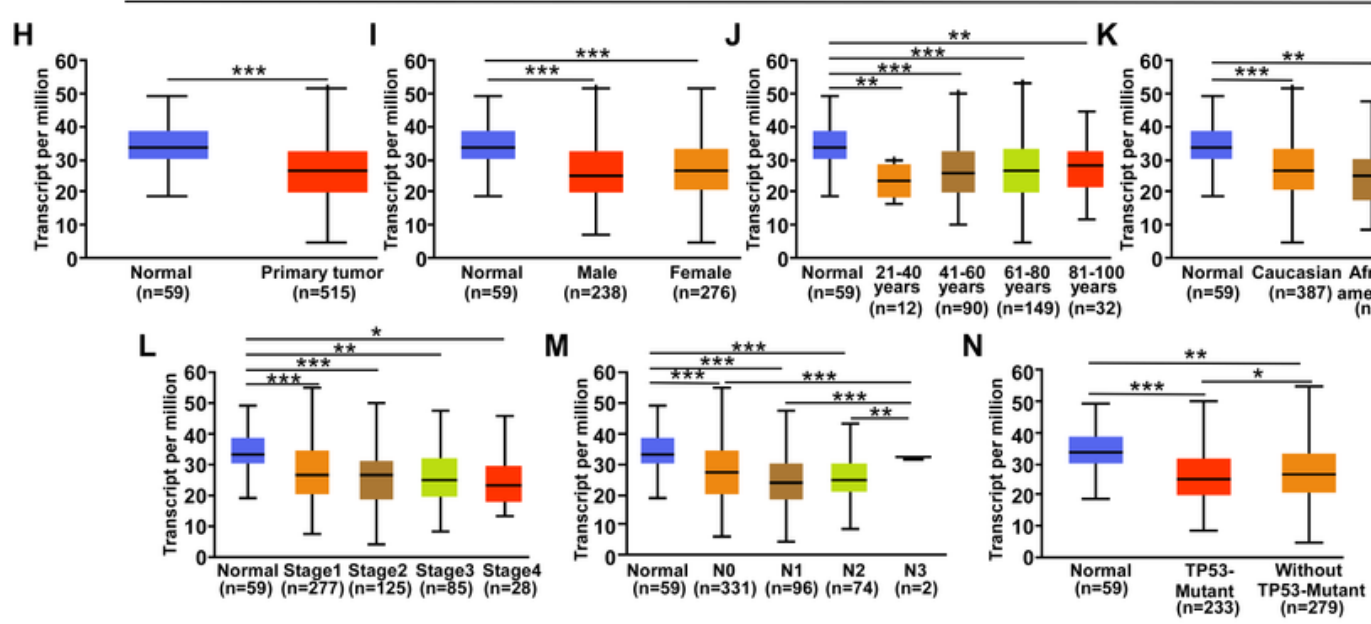

ALAD expression in LUSC
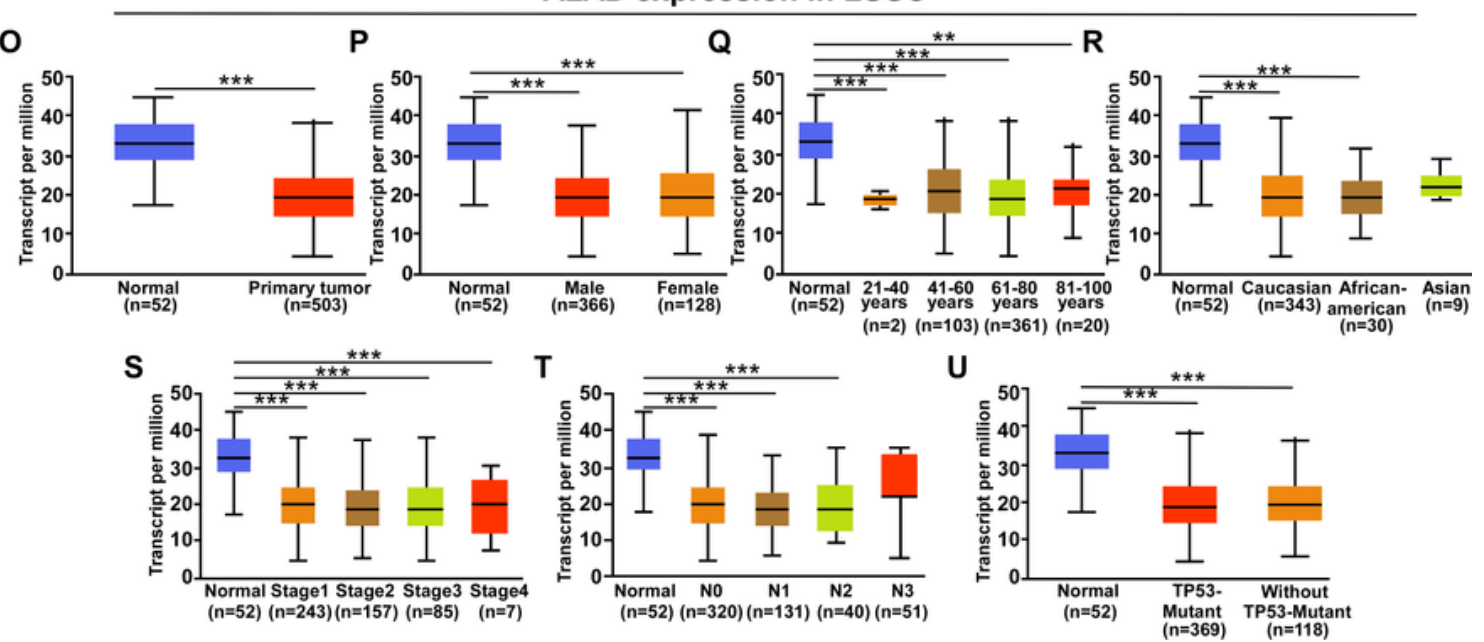

Figure 3

Subgroup expression analysis of ALAD in BRAC, LUAD, and LUSC. $(A, H, O)$ ALAD mRNA expression in cancer tissues and normal tissues in BRCA, LUAD, and LUSC patients. (B-D, I-K, P-R) ALAD mRNA expression levels in BRCA, LUAD, and LUSC patients with subgroups analysis in genders, ages, and races. $(E, L, S)$ ALAD mRNA expression levels in BRCA, LUAD, and LUSC patients with different tumor stages. (F, $\mathrm{M}, \mathrm{T}$ ) ALAD mRNA expression levels in BRCA, LUAD, and LUSC patients with different metastasis status. 
$(\mathrm{G}, \mathrm{N}, \mathrm{U})$ ALAD mRNA expression levels in BRCA, LUAD, and LUSC patients with TP-53 mutant or TP-53 non-mutant. (A-U) Graphs are generated from the UALCAN database, ${ }^{*}<<0.05$, ${ }^{*} \mathrm{P}<0.001$, $* \star * P<0.0001$.

Figure 4

A
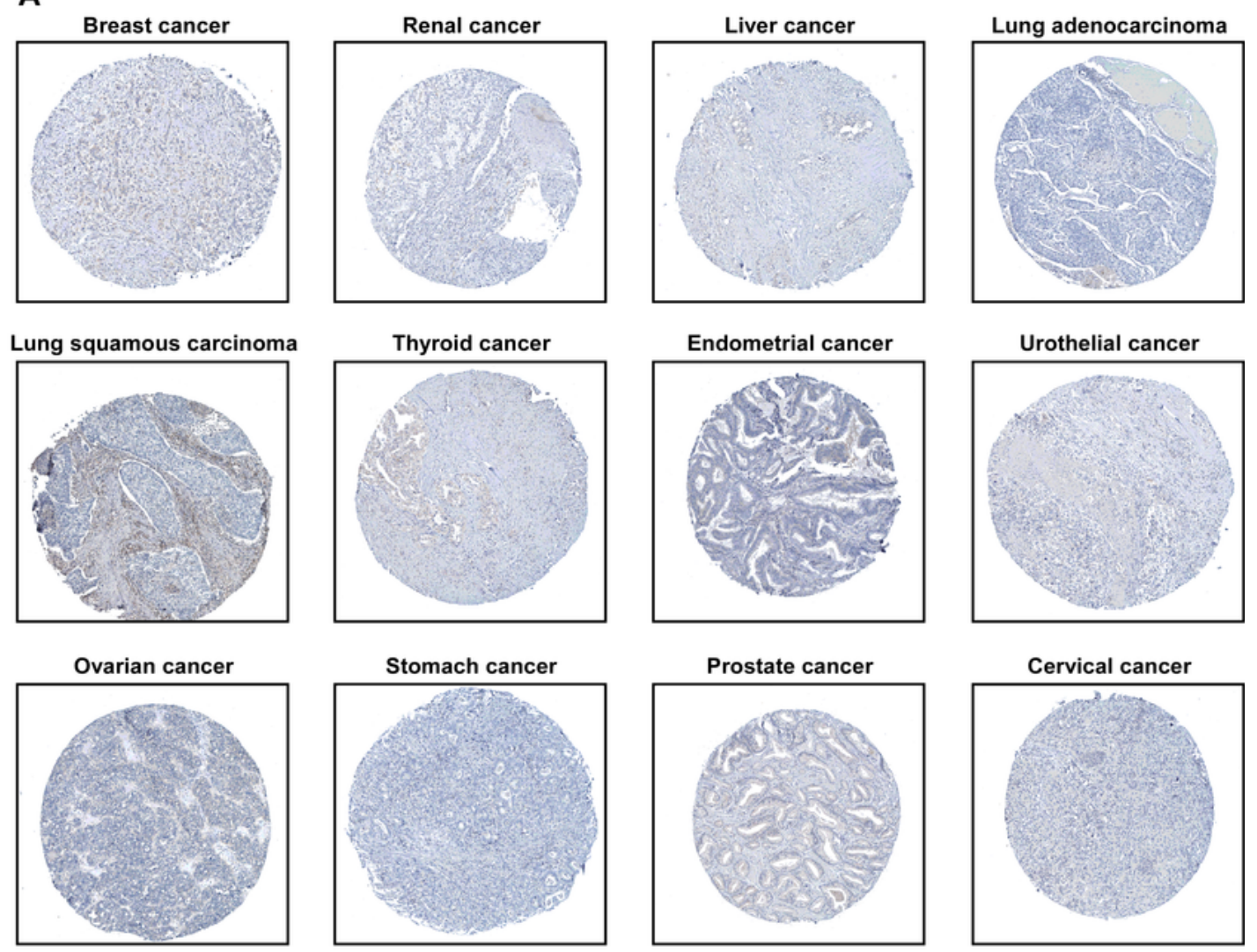

B

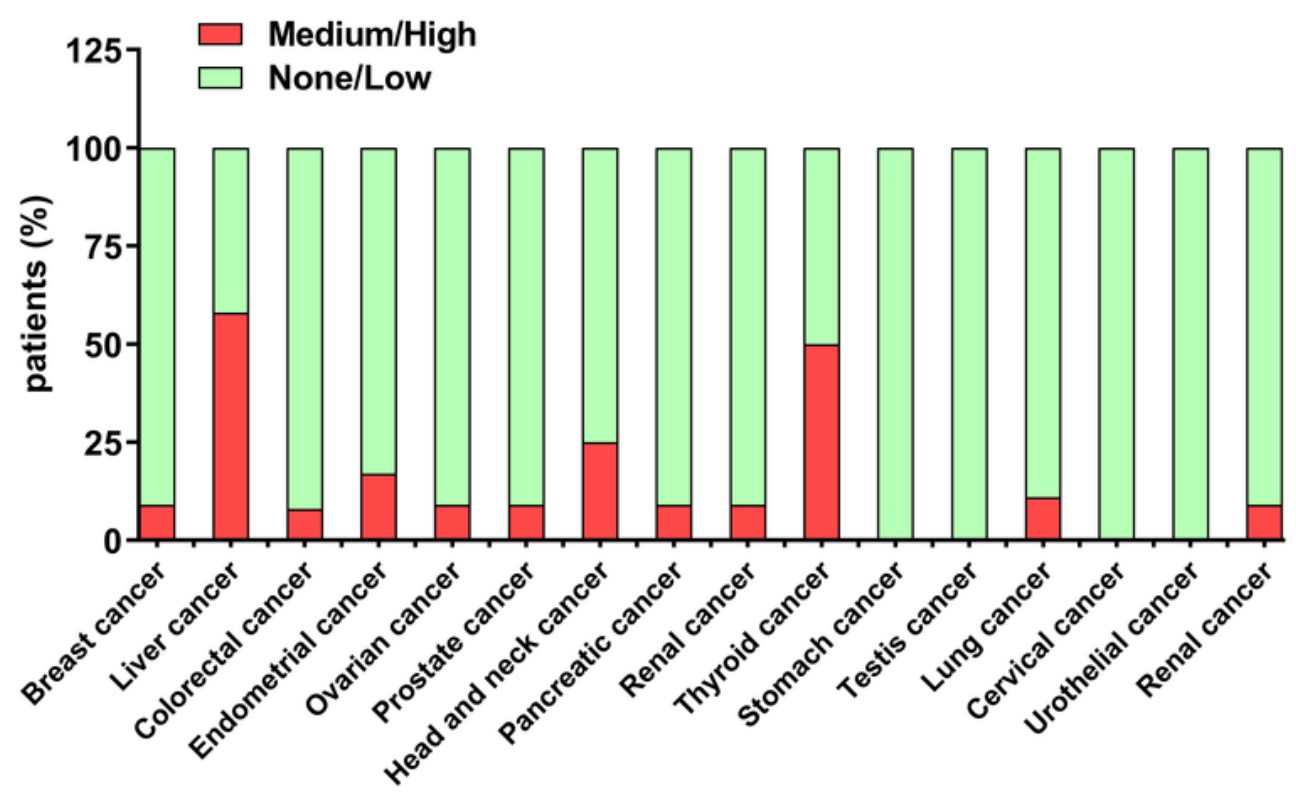

Figure 4

The expression of ALAD protein was downregulated in different solid tumors. (A) Represent pictures of the immunohistochemical staining of ALAD protein expression in different tumors in the database of the 
Human Pathological Atlas (HPA). (B) ALAD protein expression levels of 16 cancers were analyzed by IHC in the Human Pathological Atlas database (HPA).

Figure 5

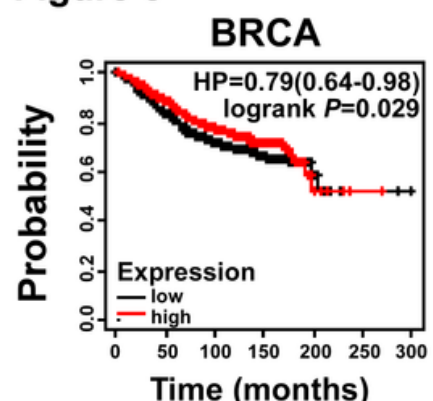

LUAD

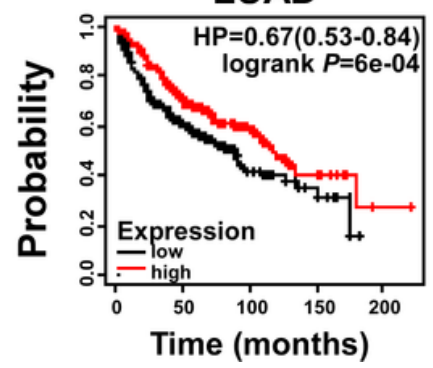

ESCA

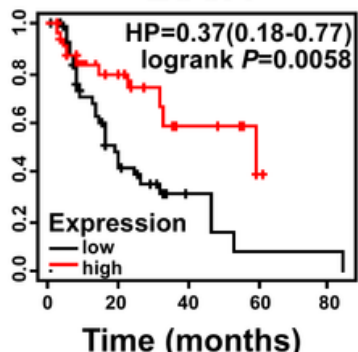

LUSC

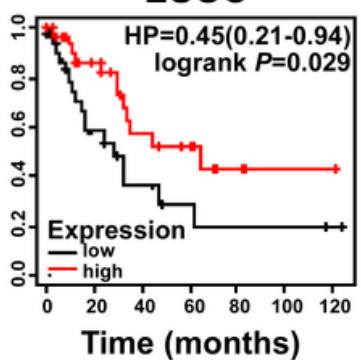

KIRC

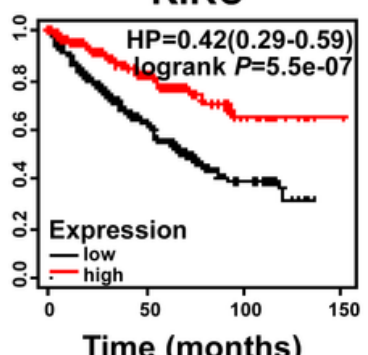

SARC

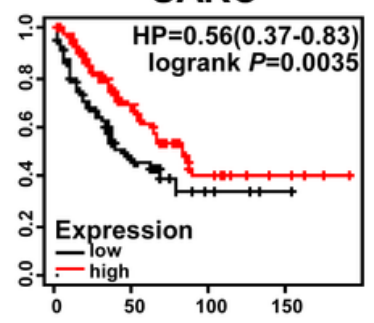

Time (months)
KIRP

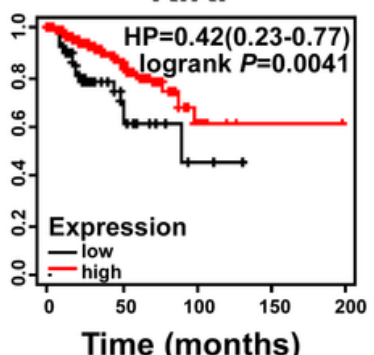

THYM

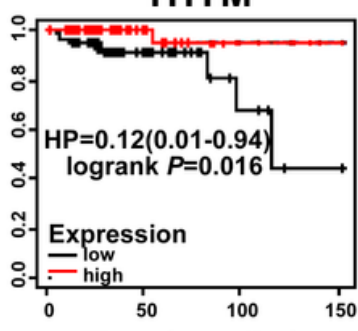

Time (months)
LIHC

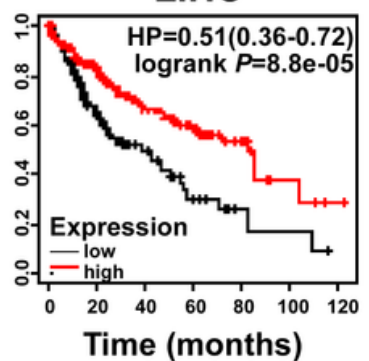

UCEC

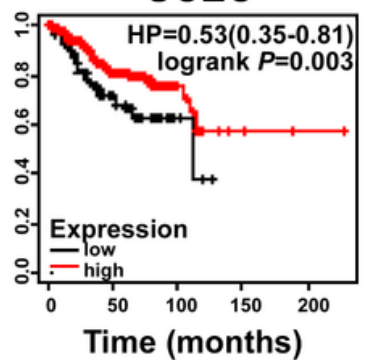

Figure 5

ALAD is a prognostic biomarker in several solid tumor types. Survival curves are plotted using KM plotter analysis for all cancer patients. Abbreviations: BRCA, Breast invasive carcinoma; ESCA, Esophageal Cancer; KIRC, Kidney renal clear cell carcinoma; KIRP, Kidney renal papillary cell carcinoma; LIHC, Liver hepatocellular carcinoma; LUAD, Lung Adenocarcinoma; LUSC, Lung squamous Cell carcinoma; SARC, Sarcoma; THYM, Thoracic Adenocarcinoma; UCEC, Uterine Corpus Endometrial Carcinoma. 

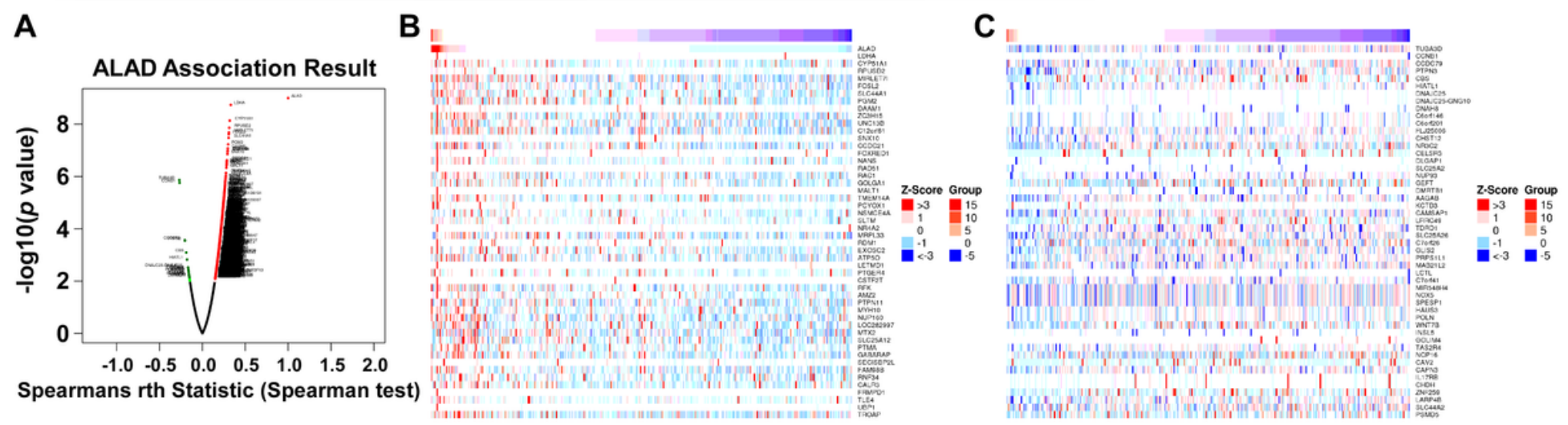

LUAD

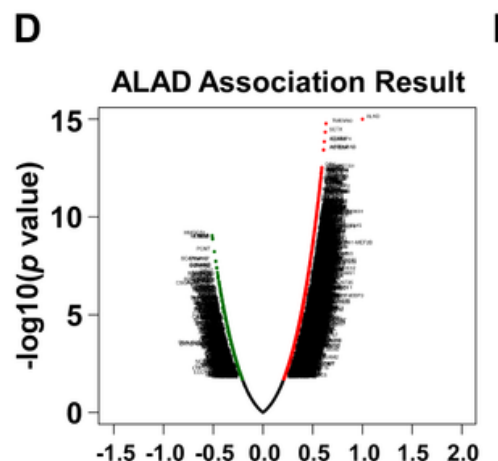

Spearmans rth Statistic (Spearman test)
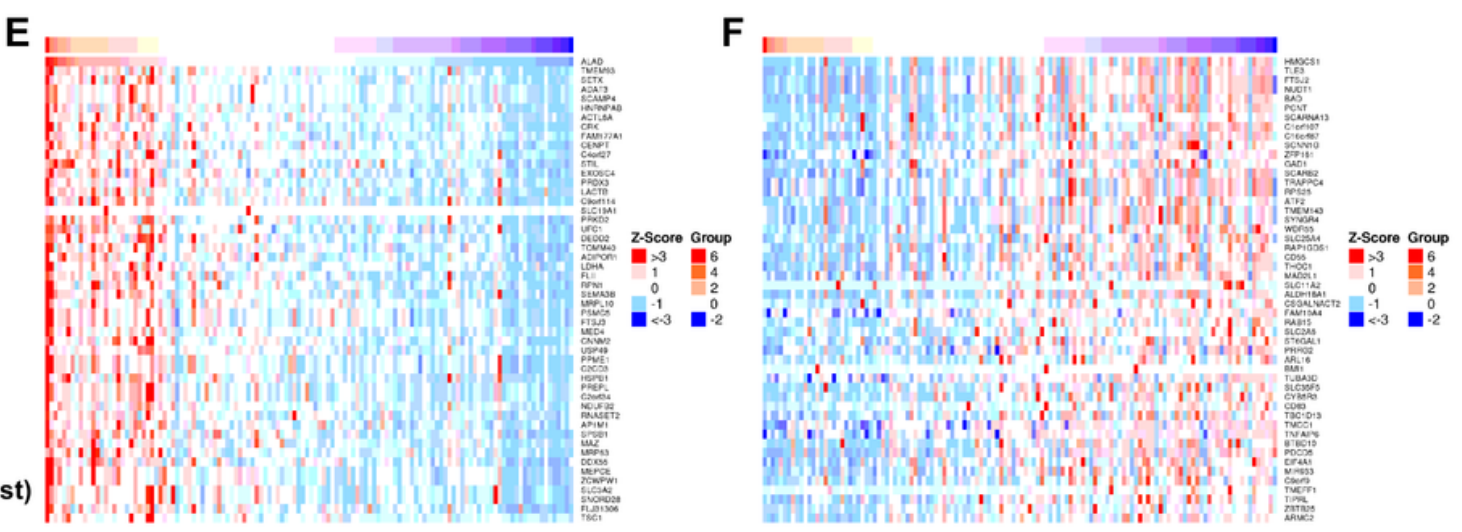

LUSC

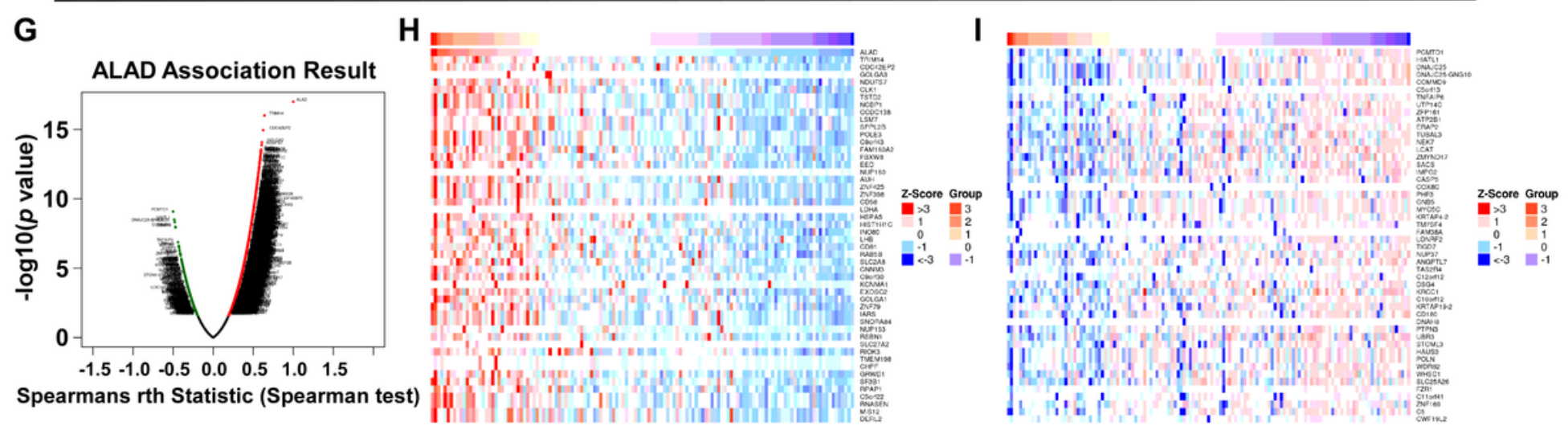

Figure 6

Differentially expressed genes (DEGs) related to ALAD in different tumors. (A) Correlation between ALAD and differentially expressed genes in BRCA (Spearman correlation analysis). (B-C) The first 50 DEGs that are positively or negatively associated with ALAD in BRCA in the heat maps. (D) Correlation between ALAD and differentially expressed genes in LUAD (Spearman correlation analysis). (E-F) The first 50 DEGs that are positively or negatively associated with ALAD in LUAD in the heat maps. (G) Correlation between ALAD and differentially expressed genes in LUSC (Spearman correlation analysis). (H-I) The first 50 DEGs that are positively or negatively associated with ALAD in LUSC in the heat maps. 
Figure 7

A

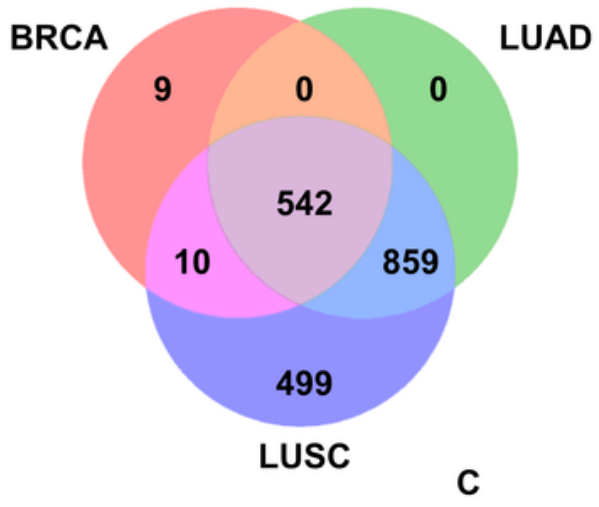

B

Biological process

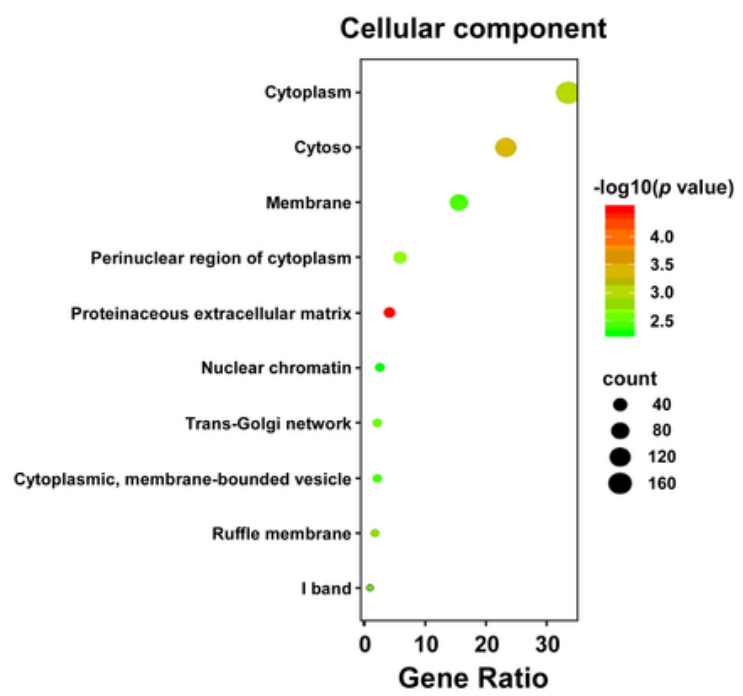

D

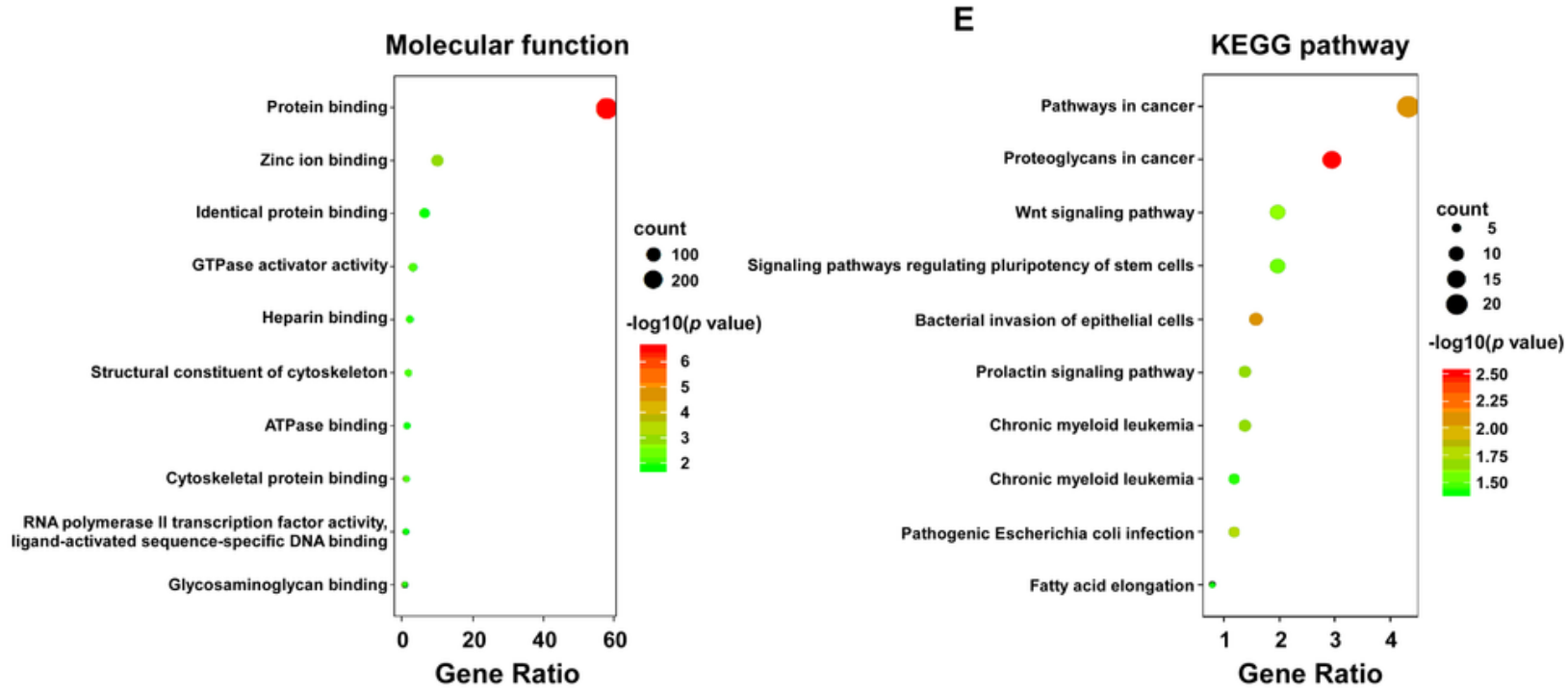

Figure 7

GO analysis and KEGG analysis about DEGs in in BRCA, LUAD and LUSC. (A) 542 differentially expressed genes (DEGs) are showed in Venn diagram. Spearman test $>0.2$ for BRCA and Spearman test $>0.4$ for LUAD and LUSC. (B) The influence of ALAD and DEGs on Biological Process (BP). (C) The aggregation of ALAD and DEGs in Cell Composition (CC). (D) The co-action of ALAD and DEGs in Molecular Function 
(MF). (E) KEGG enrichment analysis showed the co-expression signal pathways between ALAD and differentially expressed genes.

\section{Figure 8}

A

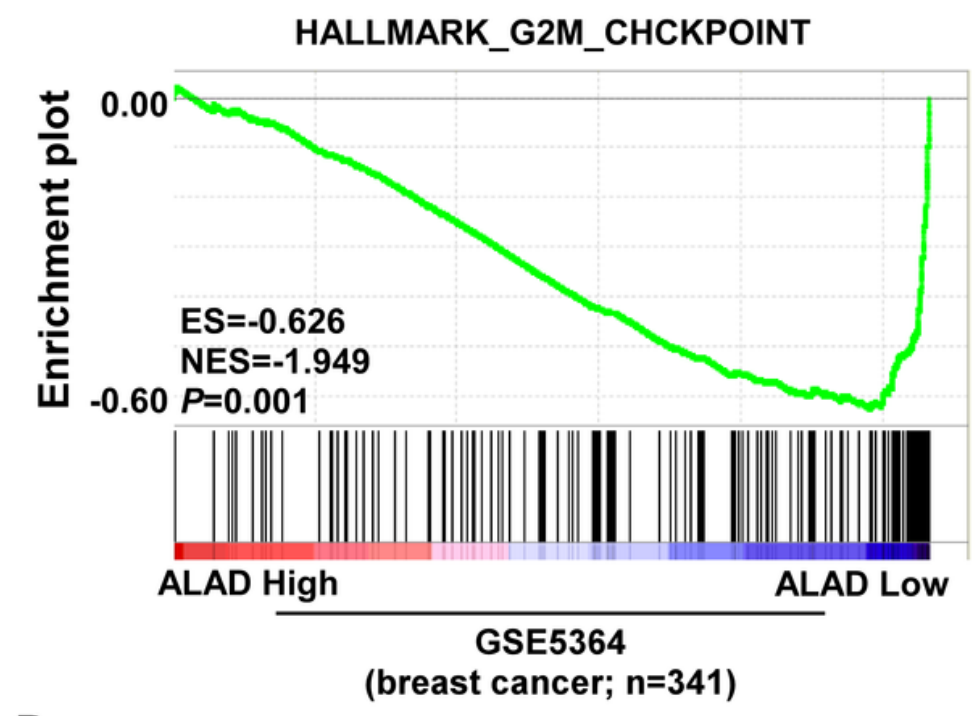

B
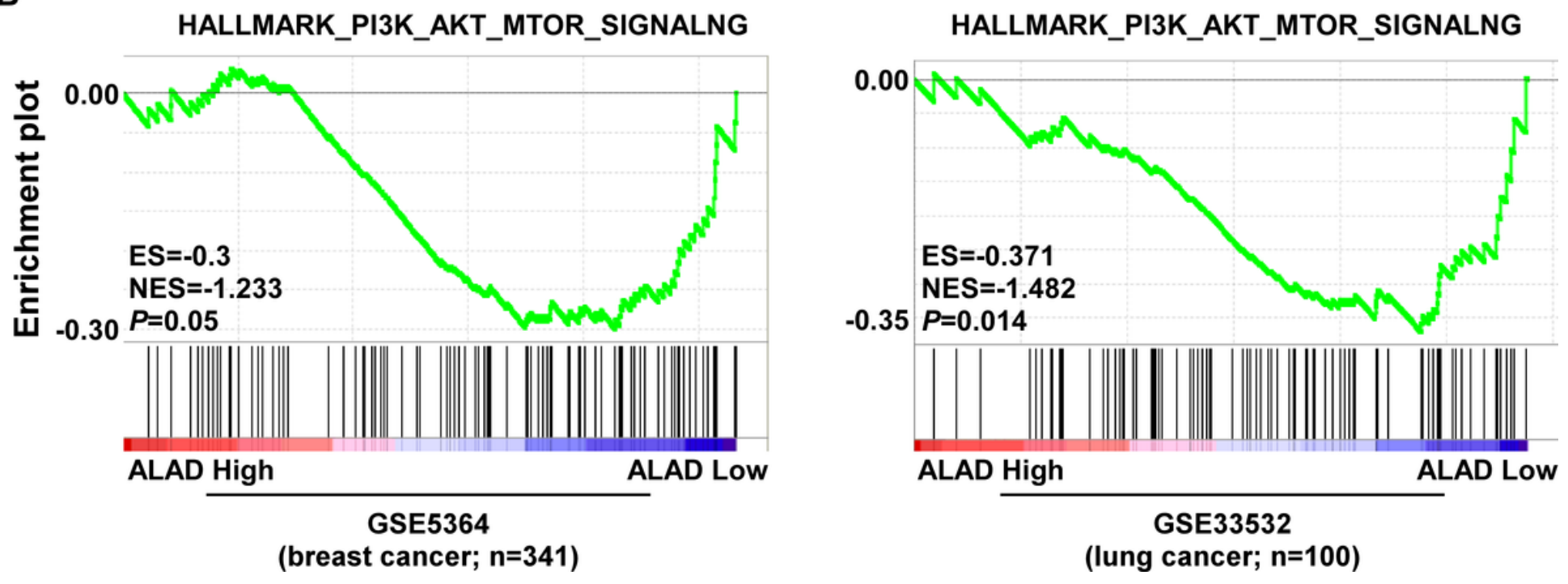

Figure 8

Overexpression of ALAD inhibits cell cycle and PI3K/AKT/mTOR signal pathway. (A) Overexpression of $A L A D$ inhibits the cell cycle of breast cancer and lung cancer. In the published gene expression profiles of breast cancer patients (NCBI/GEO/GSE5364, n=341) and lung cancer patients (NCBI/GEO/GSE33532, $\mathrm{n}=100)$, GSEA maps showed that the expression of ALAD was negatively correlated with cell cycle signal (HALLMARK_G2M_CHCKPOINT). (B) Overexpression of ALAD inhibits the PI3K/AKT/mTOR signal pathway. In the published gene expression profiles of breast cancer patients (NCBI/GEO/GSE5364, $\mathrm{n}=341$ ) and lung cancer patients (NCBI/GEO/GSE33532, n=100), GSEA results showed that the expression of ALAD was negatively correlated with the PI3K/AKT/mTOR pathway activation (HALLMARK_PI3K_AKT_MTOR_SIGNALNG). 